\title{
Molecular characterization of a Trichinella spiralis enolase and its interaction with the host's plasminogen
}

\author{
Peng Jiang, You Jiao Zao, Shu Wei Yan, Yan Yan Song, Dong Min Yang, Li Yuan Dai, Ruo Dan Liu, Xi Zhang, \\ Zhong Quan Wang ${ }^{*}$ and Jing Cui ${ }^{*}$
}

\begin{abstract}
The binding and activation of host plasminogen (PLG) by worm surface enolases has been verified to participate in parasite invasion, but the role of this processes during Trichinella spiralis infection has not been clarified. Therefore, the expression and immunolocalization of a T. spiralis enolase (TsENO) and its binding activity with PLG were evaluated in this study. Based on the three-dimensional (3D) molecular model of TsENO, the protein interaction between TsENO and human PLG was analysed by the ZDOCK server. The interacting residues were identified after analysis of the protein-protein interface by bioinformatics techniques. The key interacting residues were confirmed by a series of experiments. The GPCR analysis results demonstrated that Ts-eno was transcribed throughout the whole life cycle of T. spiralis. The immunofluorescence assay (IFA) results confirmed that TSENO was distributed on the T. spiralis surface. The binding assays showed that recombinant TsENO (rTSENO) and native TsENO were able to bind PLG. Four lysine residues $(90,289,291$ and 300) of TsENO were considered to be active residues for PLG interaction. The quadruple mutant (Lys90Ala + Lys289Ala + Lys291 Ala + Lys300Ala) TsENO, in which the key lysine residues were substituted with alanine (Ala) residues, exhibited a reduction in PLG binding of nearly 50\% (45.37\%). These results revealed that TsENO has strong binding activity with human PLG. The four lysine residues (90, 289, 291 and 300) of TsENO play an important role in PLG binding and could accelerate PLG activation and invasion of the host's intestinal wall by T. spiralis.
\end{abstract}

\section{Introduction}

Plasminogen (PLG) circulates throughout the endovascular network and serves as a zymogen that initiates the process of fibrinolysis [1, 2]. PLG is synthesized by several organs/tissues, mainly the liver and simultaneously by the kidney, brain, heart, lung, spleen, and gut; in addition, a considerable quantity is found in extravascular fluids $[3,4]$. The critical and final step in the process of fibrinolytic system activation is PLG activation; this activation is usually dependent on activators that convert PLG into its active form, plasmin (PLM) [5-8]. PLG and PLM play fundamental roles in fibrinolysis and the degradation of structures related to tissue repair and immunity, such as fibrin clots and the extracellular matrix

*Correspondence: wangzq2015@126.com; cuij@zzu.edu.cn Department of Parasitology, Medical College, Zhengzhou University, Zhengzhou 450052, China
(ECM) $[8,9]$. Evidently, the activation of PLG is utilized by some pathogens to penetrate the tissue barrier and facilitate invasion [10].

The interaction between PLG and pathogens is usually regulated by various and ubiquitous PLG receptors [1113]. A fairly large number of pathogens, including bacteria, fungi and parasites, exhibit surface expression of PLG receptors that immobilize PLG, resulting in its activation; it has been proposed that the activation of PLG facilitates the migration and invasion of these pathogens to different tissues in the host [14-17]. To date, more than 85 different kinds of PLG receptors have been identified to play roles in infectious diseases [18]. Among these receptors, enolase is perhaps the most studied PLG-binding protein in different organisms and is frequently exploited by parasites [18-21]. Enolase is not only a glycolytic enzyme but also a multifunctional protein [20]. As a

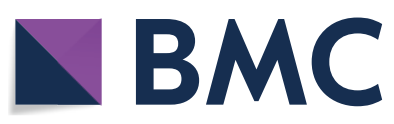

(c) The Author(s) 2019. This article is distributed under the terms of the Creative Commons Attribution 4.0 International License (http://creativecommons.org/licenses/by/4.0/), which permits unrestricted use, distribution, and reproduction in any medium, provided you give appropriate credit to the original author(s) and the source, provide a link to the Creative Commons license, and indicate if changes were made. The Creative Commons Public Domain Dedication waiver (http://creativecommons.org/ publicdomain/zero/1.0/) applies to the data made available in this article, unless otherwise stated. 
surface-expressed PLG receptor, enolase is able to mediate the activation of PLG and the degradation of the ECM [21].

Trichinellosis is an important food-borne zoonosis caused by the ingestion of raw or undercooked meat contaminated with viable, infective $T$. spiralis larvae. The critical variable in the establishment of $T$. spiralis infection is whether the larvae invade the intestinal mucosa to develop further. However, the mechanism of larval invasion is unclear to date [22-24]. Our previous LC-MS/MS results of infective larval proteins showed that $T$. spiralis enolase (TsENO) was present at very high levels after coculture with intestinal epithelial cells (IECs) in vitro [25]. The relative transcription level of the $T$. spiralis enolase gene (Ts-eno) was upregulated in larvae cultured with IECs [26]. High expression of TsENO was also identified in a screen of $T$. spiralis intestinal infective larvae (IIL) surface proteins [27].

Previous studies indicate that TsENO may bind the host's PLG to activate the fibrinolytic system, degrade the ECM, and promote larval penetration of the tissue barrier during invasion. For the purpose of further understanding the relationship between T. spiralis and its host, bioinformatics methods and experimental techniques were used to explore the interaction between TsENO and the host's PLG in this work.

\section{Materials and methods \\ Bioinformatics analysis, molecular modelling and model evaluation of TsENO}

The whole coding cDNA sequence (CDS) of TsENO (Tsp_09466) was retrieved from GenBank (Accession no. XM_003371185). The three-dimensional (3D) molecular structure of full-length human PLG (X-ray crystal structure; ID: 4DUR) was obtained from the Protein Data Bank (PDB). The background biochemical characteristics of TsENO were analysed by bioinformatics software and web servers. Physical and chemical parameters of the TsENO protein were predicted with the ProtParam tool [28]. SignalP Server (version 4.1) was used to predict the cleavage sites in the signal peptide [29]. A molecular model of TsENO was generated by the threading protein server Iterative Threading ASSEmbly Refinement (I-TASSER) [30]. The 3D structure and the quality of the models were evaluated and verified by SAVES v5.0 [31-35].

\section{Protein-protein docking}

The ZDOCK (version 3.0.2) algorithm with the default parameters was used to predict the structures of the TsENO-PLG complex. ZDOCK was used to search and analyse all possible binding poses between TsENO and PLG in 3D space and to assess every binding pose by an energy-based scoring function [36]. The docking results were visualized by visual molecular dynamics (VMD) [37].

\section{Analyses of protein-protein docking}

TsENO-PLG interaction plots were generated by the DIMPLOT algorithm in LigPlot+ (version 2.1) [38]. To check the involvement of TsENO-interacting residues obtained from DIMPLOT, the H-bonding and hydrophobic interactions between TsENO and PLG and the loss of accessible surface area (ASA) and solvation energy $\left(\Delta^{\mathrm{i}} G\right)$ of the interfacing residues were re-checked and confirmed by PDBe PISA (version 1.52) [39]. 3D structural alignments between TsENO and its variants were generated by SuperPose (version 1.0) and Swiss-PdbViewer (DeepView, version 4.1) [40, 41].

\section{Expression and purification of TsENO and its site-directed mutant}

The coding sequences for TsENO and its site-specific mutant sequence were chemically synthesized by Sangon Biotech Co., Ltd. (Shanghai, China) and introduced into Escherichia coli. The synthesized coding sequences harbouring BamHI and PstI restriction enzyme sites were cloned into the prokaryotic expression vector pQE-80L with one step cloning kit (Vazyme, Nanjing, China). Recombinant $\mathrm{pQE}-80 \mathrm{~L} / \mathrm{TsENO}$ and its variant were transformed into E. coli BL21 (DE3) (Novagen, La Jolla, USA). The expression of rTsENO and its mutant (M-rTsENO) was induced for $5 \mathrm{~h}$ at $30{ }^{\circ} \mathrm{C}$ by using $0.5 \mathrm{mM}$ isopropyl $\beta$-D-1-thiogalactopyranoside (IPTG). The rTsENO and M-rTsENO proteins were purified using a Ni-NTA sefinose ${ }^{\mathrm{TM}}$ resin kit (Sangon Biotech, Shanghai, China). The concentrations of the purified proteins were measured by the bicinchoninic acid (BCA) method, and the recombinant proteins were then analysed by SDSPAGE with $12 \%$ acrylamide separating gels.

\section{Preparation of anti-rTsENO and M-rTsENO antibodies}

Fifteen BALB/c mice (female, 6 weeks old, purchased from the Experimental Animal Center of Henan Province) were vaccinated with $r T s E N O$. The rTsENO protein was emulsified in Freund's complete adjuvant and was subcutaneously injected into the abdomen of the BALB/c mice ( $20 \mu \mathrm{g}$ rTsENO per mouse). All mice were boosted twice by using the same amount of rTsENO (emulsified in Freund's incomplete adjuvant) at intervals of 10 days. Blood samples ( $50 \mu \mathrm{L} /$ mouse) from vaccinated mice were collected 10 days after the final immunization. The antirTsENO antibody titre in serum was measured by ELISA. The serum anti-mutated rTsENO antibody titre was measured by the same protocol. 


\section{Real-time quantitative PCR (qPCR) analysis}

The T. spiralis isolate (ISS534) used in this study was maintained in BALB/c mice by oral inoculation in our laboratory. Total RNA of T. spiralis muscle larvae (ML), IIL, 3-day-old adult worms (3 days AW), 6 days AW and newborn larvae (NBL) were extracted by Trizol (Invitrogen $^{\mathrm{TM}}$, Carlsbad, USA), and the RNA quality was assessed by agarose gel electrophoresis and ultramicrospectrophotometry (NanoDrop 2000, Thermo Scientific, Wilmington, USA). Then, RNA was transcribed to cDNA by using an RNA PrimeScript ${ }^{\mathrm{TM}}$ RT reagent kit containing DNase I (with gDNA Eraser, Takara, Japan), which can digest single- and double-stranded DNA [42]. Primer Premier 5 was used to design the primers for Ts-eno $\left(5^{\prime}-\right.$ AAACGGCGGTTCTCACGCAG-3'; ${ }^{\prime}$ '-TCGGCGCAA ATCCACCTTCG-3'). The housekeeping gene glyceraldehyde-3-phosphate dehydrogenase (GAPDH) was used as the standard control (5'-AGATGCTCCTATGTTGGT TATGGG-3'; 5'-GTCTTTTGGGTTGCCGTTGTAG$\left.3^{\prime}\right)$. Fifty nanograms of cDNA in a $20 \mu \mathrm{L}$ reaction volume was used to quantify the transcriptional level of Ts-eno by using a 7500 Fast Real-Time PCR System (Applied Biosystems, Foster City, USA) as previously described $[23,26]$. All experiments were repeated three times. The two amplicons of Ts-eno and GAPDH were examined by $2 \%$ agarose gel electrophoresis. The melt curves of Ts-eno and GAPDH were generated by 7500 Software (version 2.0.5 for 7500 Fast Real-Time PCR Products, Applied Biosystems, USA). The comparative Ct method $\left(2^{-\Delta \Delta C t}\right)$ was used to determine the normalized transcription level of Ts-eno $[25,43]$.

\section{Immunolocalization of TsENO}

An indirect immunofluorescence assay (IFA) was used to identify whether TsENO was expressed on the surface of T. spiralis worms at different stages (ML, $6 \mathrm{~h} \mathrm{IIL,} 24 \mathrm{~h}$ IIL, 3 days AW, 6 days AW and NBL) as previously described $[44,45]$. Whole worms were blocked in $5 \%$ goat serum diluted with PBS for $1 \mathrm{~h}$ and were then incubated with a 1:10 dilution of anti-rTsENO serum in a moist chamber at $37^{\circ} \mathrm{C}$ for $1 \mathrm{~h}$. At the same time, serum from T. spiralisinfected mice and normal mouse serum were utilized as the positive control and negative control, respectively. After three rinses with PBS, worms were incubated with FITC-labelled goat anti-mouse IgG (Santa Cruz, USA) diluted 1:100 for $1 \mathrm{~h}$ at $37^{\circ} \mathrm{C}$. Following five washes in $\mathrm{PBS}$, the parasites were finally examined under a fluorescence microscope (Olympus, Japan).

\section{Analysis of PLG binding by Western blotting}

For Western blot analysis, purified rTsENO, purified M-rTsENO, ML soluble antigens, ML excretory-secretory
(ES) antigens, S. cerevisiae enolase (ScENO; positive control; Sigma-Aldrich, Saint Louis, USA) and BSA (negative control) were loaded into the lanes of a 12\% SDS-PAGE gel and electrophoresed. Then, the proteins were transferred to an NC membrane (Millipore, USA), which was blocked with $5 \%$ skim milk in TBST at room temperature for $2 \mathrm{~h}$. After washing three times with TBST, the membrane was then incubated with $25 \mu \mathrm{g} / \mathrm{mL}$ human PLG (Sigma-Aldrich) in PBST at $37^{\circ} \mathrm{C}$ for $3 \mathrm{~h}$, washed three times with TBST, and incubated with a sheep antihuman PLG antibody (1:2500; Invitrogen $\left.{ }^{\mathrm{TM}}\right)$ in TBST for $2 \mathrm{~h}$ at room temperature. After washing with TBST, the membrane was incubated with rabbit anti-sheep IgGHRP (1:10 000; Invitrogen ${ }^{\text {TM }}$ ) for $2 \mathrm{~h}$ at room temperature. After washing three times with TBST, the strips were developed using the 3-amino-9-ethylcarbazole (AEC; Solarbio, Beijing, China) substrate.

\section{Analysis of PLG binding by indirect ELISA}

To compare the PLG binding ability between rTsENO and M-rTsENO, an ELISA plate binding assay was adopted. In brief, the ELISA plate was coated with $0.6 \mu \mathrm{g} /$ well purified rTsENO and incubated overnight at $4{ }^{\circ} \mathrm{C}$. After being washed with PBST three times, the plate was blocked with $5 \%$ skim milk for $2 \mathrm{~h}$ at $37^{\circ} \mathrm{C}$. The plate was washed three times again, and increasing concentrations of human PLG $(0.05,0.1,0.2,0.4,0.8,1.2,1.6$, and $2.0 \mu \mathrm{g} /$ $\mathrm{mL}$ in PBST) were then added in duplicate and incubated for $3 \mathrm{~h}$ at $37^{\circ} \mathrm{C}$. After washing, the plates were incubated with a sheep anti-human PLG antibody (1:2500; $100 \mu \mathrm{L} /$ well) at $37^{\circ} \mathrm{C}$ for $2 \mathrm{~h}$. After washing, rabbit anti-sheep IgG-HRP was added to the wells $(1: 10000 ; 100 \mu \mathrm{L} /$ well), and the plates were incubated at $37{ }^{\circ} \mathrm{C}$ for $1 \mathrm{~h}$. Finally, the compound o-phenylenediamine dihydrochloride (OPD; Sigma-Aldrich) was used as the substrate for HRP. The optical density (OD) at $450 \mathrm{~nm}$ was measured in an ELISA reader (Tecan Schweiz AG, Mannedorf, Switzerland). The wells coated with M-rTsENO $(0.6 \mu \mathrm{g} /$ well $)$, ScENO $(0.4 \mu \mathrm{g} /$ well, positive control $)$ and BSA $(0.8 \mu \mathrm{g} /$ well, negative control) were analysed by the same procedures. All experiments in this part were repeated three times.

\section{Statistical analysis}

The data were analysed by IBM SPSS Statistics 21.0 for Windows (IBM Corporation, NY, USA). The differences in the relative Ts-eno expression levels across the different stages were analysed with one-way ANOVA. The intra- and inter-group differences in OD values were assessed by Student's $t$ test and one-way ANOVA as appropriate. The variation trend in the OD values with increasing PLG concentrations was evaluated by a linear 
algorithm in an ANOVA model. A significance level of $P<0.05$ was regarded as statistically significant.

\section{Results}

Biochemical characteristics and molecular model of TsENO

The complete CDS of T. spiralis enolase (XM_003371185) contains $1461 \mathrm{bp}$ and encodes 486 amino acids (aa). The SignalP 4.1 prediction showed that there is a signal peptide with a cleavage site between the aa at pos. 13 and 14. The length of the mature protein of TsENO is 473 aa; the molecular mass is $51.95 \mathrm{kDa}$, and the theoretical isoelectric point (pI) is 5.88. For the aa sequence of TsENO, the I-TASSER server generated and reported up to five models that correspond to the states with the largest partition function (or lowest free energy). Among the top 5 models, the best model, with the highest C-score, was selected as the final molecular model of TsENO (Figure 1). The selected TsENO model was further evaluated by the SAVES v5.0 program. According to the Verify 3D results, $82.24 \%$ of the residues had a score of $\geq 0.2$ in the 3D-1D profile. The overall quality factor was 90.753 by the ERRAT results. The model quality also passed the PROVE, WHATCHECK and PROCHECK evaluations. The Ramachandran plot revealed that $78.1 \%$ of

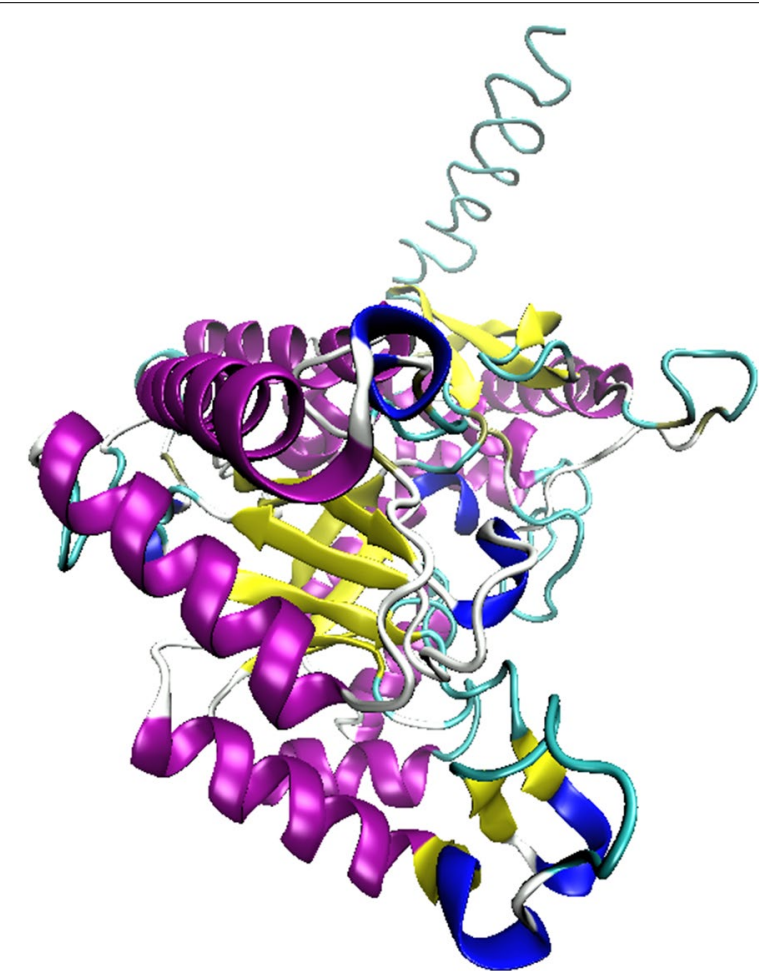

Figure 1 Cartoon representation of TsENO molecular model. Secondary structures are coloured differently, with alpha helices in purple, 3_10_helices in blue, extended beta strands in yellow, bridge beta strands in tan, coils in white, and turns in cyan. the TsENO residues were in the most favoured region, $20.7 \%$ were in allowed regions, and only $1.2 \%$ were in disallowed regions, suggesting the validity of the TsENO model (Figure 2).

\section{Molecular docking between TsENO and PLG}

Via the ZDOCK program, every possible TsENO-PLG binding pose in 3D space calculated and evaluated, and each pose was scored using an energy-based scoring function. The results of the top 2000 binding poses were filtered using a 6 angstrom $(\AA)$ distance cutoff for the interacting residues. The reserved predictions were sorted by the ZDOCK score, and the top prediction was selected and further analysed. The ZDOCK proteinprotein docking results indicated that TsENO and PLG exhibited surface complementarity in the interface area (Figure 3).

\section{PLG-interacting residues of TsENO}

The hydrophobic effect and $\mathrm{H}$-bond interaction between TsENO and PLG were analysed by PDBe PISA v1.52 and DIMPLOT. The changes in the accessible surface area (ASA) and the solvation energy effect $\left(\Delta^{\mathrm{i}} \mathrm{G}\right)$ were calculated by PDBe PISA. Seven lysine residues (90, 198, 229, 233, 289, 291 and 300) were identified in the interface area, and lysine was deemed to be the most frequently appearing interacting amino acid (Table 1, Figure 4A). Lys90 and Lys198 formed H-bonds with PLG, but the

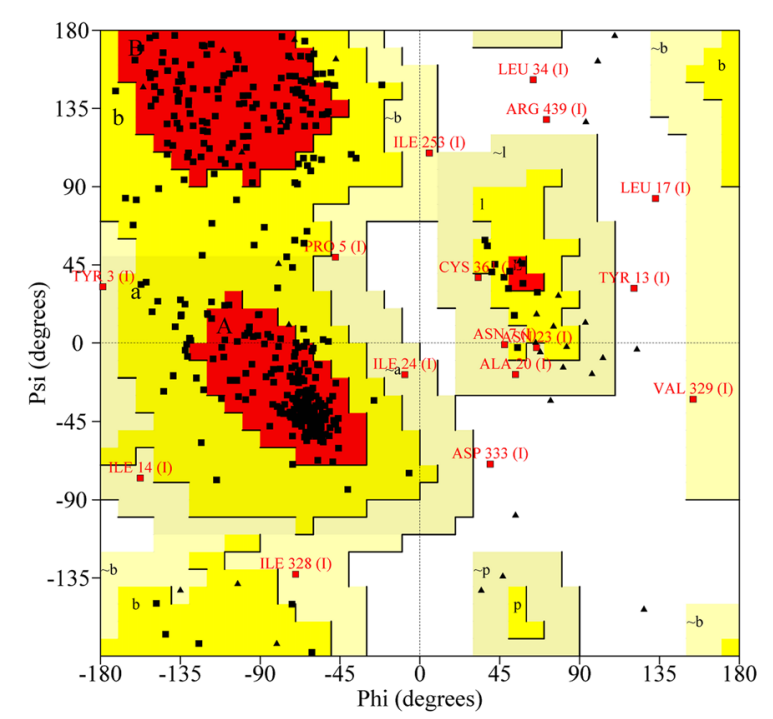

Figure 2 Ramachandran plot analysis of the TsENO molecular model. All residues except Gly and Pro are shown as square dots located in the most favoured regions (78.1\% in the red area), additional allowed regions (18.3\% in the yellow area), generously allowed regions ( $2.4 \%$ in the yellow-grey area) and disallowed regions $(1.2 \%$ in the white area). 


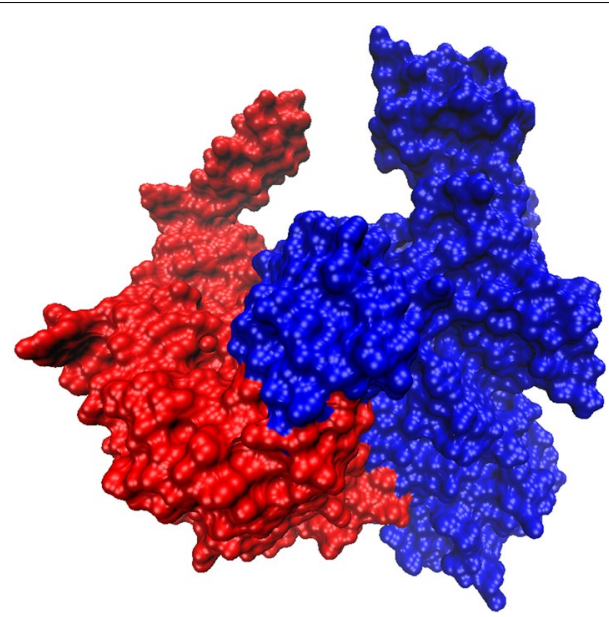

Figure 3 Protein-protein docking of TsENO and human PLG. Binding proteins are shown as surface representations. TsENO is shown in red, whereas human PLG (4DUR) is shown in blue.

ASA loss for Lys198 was only $6.95 \AA^{2}$. The interface showed that these lysine residues, except Lys198, had hydrophobic interactions with PLG. According to the simulated calculation, after 4 of the interacting lysine residues $(90,289,291$ and 300) were substituted with alanine (Ala) residues, all of the lysine residues did not participate in the TsENO-PLG surface interaction. These four lysine residues (90, 289, 291 and 300) of TsENO were identified as active residues for PLG binding (Table 1, Figures 4B and $\mathrm{C}$ ). The molecular model of the quadruple mutant (Lys90Ala + Lys289Ala + Lys291Ala + Lys300Ala) TsENO was also constructed by I-TASSER, and 3D structural alignments between TsENO and its quadruple mutant were performed by SuperPose and Swiss-PdbViewer. Two molecules exhibited similar 3D structures, and the local RMSD between the backbones and the global RMSD were $2.63 \AA$ and $3.30 \AA$, respectively (Figure 5).

\section{Expression and purification of rTsENO and M-rTsENO}

The synthesized coding sequences of TsENO and $\mathrm{M}$-TsENO were successfully cloned into the pQE-80L vector. E. coli BL21 harbouring $\mathrm{pQE}-80 \mathrm{~L} / \mathrm{TsENO}$ and pQE-80L/M-TsENO were induced for $5 \mathrm{~h}$ with $0.5 \mathrm{mM}$ IPTG at $30{ }^{\circ} \mathrm{C}$. The molecular weights of rTsENO and M-rTsENO were identified by SDS-PAGE as $51.95 \mathrm{kDa}$ and $51.78 \mathrm{kDa}$, consistent with the previous predictions of ProtParam (Figure 6).

\section{Transcription levels of Ts-eno in different stages of $T$. spiralis development}

qPCR analysis was carried out to quantify the relative transcriptional level of Ts-eno across different stages of T. spiralis development (ML, IIL, 3 days AW, 6 days AW and NBL). The qPCR results revealed that Ts-eno transcription was observed at all stages of $T$. spiralis development. The $2 \%$ agarose gel electrophoresis results revealed that the Ts-eno and GAPDH amplicons had sizes of $188 \mathrm{bp}$ and $196 \mathrm{bp}$, respectively. The melt curves of Tseno and GAPDH generated by 7500 Software (version 2.0.5) showed only one peak for each amplicon, which indicated that the PCR products possessed very high specificity. The Ts-eno relative transcriptional level in ML was significantly higher than that in other developmental stages (IIL, 3 days AW, 6 days AW and NBL) $(F=7.878$, $P<0.05)$ (Figure 7).

\section{Immunolocalization of TsENO}

IFA of intact $T$. spiralis worms detected by anti-rTsENO serum showed that bright green immunofluorescence staining was observed on the epicuticles of $T$. spiralis in all life cycle stages. There was bright fluorescence staining on the cuticles of ML, $6 \mathrm{~h}$ IIL, $24 \mathrm{~h}$ IIL, 3 days AW, 6 days AW, and NBL, as probed by anti-rTsENO serum, whereas no staining was detected on the cuticles of ML incubated with normal mouse serum and PBS (Figure 8).

\section{Western blot validation of the PLG binding and interacting} residues

For Western blot analysis, the same amount of purified rTsENO and M-rTsENO, as well as ScENO, ML soluble antigens and ML ES antigens, were separated

Table 1 Shortlisted human PLG-interacting lysine residues of TsENO

\begin{tabular}{lllcrc}
\hline Lysine residue & Hydrophobic effect & H-bond interaction & ASA $\left(\AA^{\mathbf{2}}\right)$ & Loss in ASA $\left(\AA^{\mathbf{2}}\right)$ & $\boldsymbol{\Delta}^{\mathbf{i} G}(\mathbf{k c a l} / \mathbf{m o l})$ \\
\hline 90 & 3 & 1 & 146.93 & 135.98 & 1.16 \\
198 & 0 & 1 & 31.36 & 6.95 & 0.06 \\
229 & 1 & 0 & 35.26 & 35.09 & 0.56 \\
233 & 2 & 0 & 75.59 & 46.97 & 0.72 \\
289 & 2 & 0 & 53.70 & 45.08 & 0.72 \\
291 & 2 & 0 & 169.68 & 82.02 & 1.07 \\
300 & 1 & 0 & 50.51 & 45.66 & 0.31
\end{tabular}

$A S A$ accessible surface area $\left(\AA^{2}\right), \Delta i G$ solvation energy of the corresponding residue $(\mathrm{kcal} / \mathrm{mol})$. 

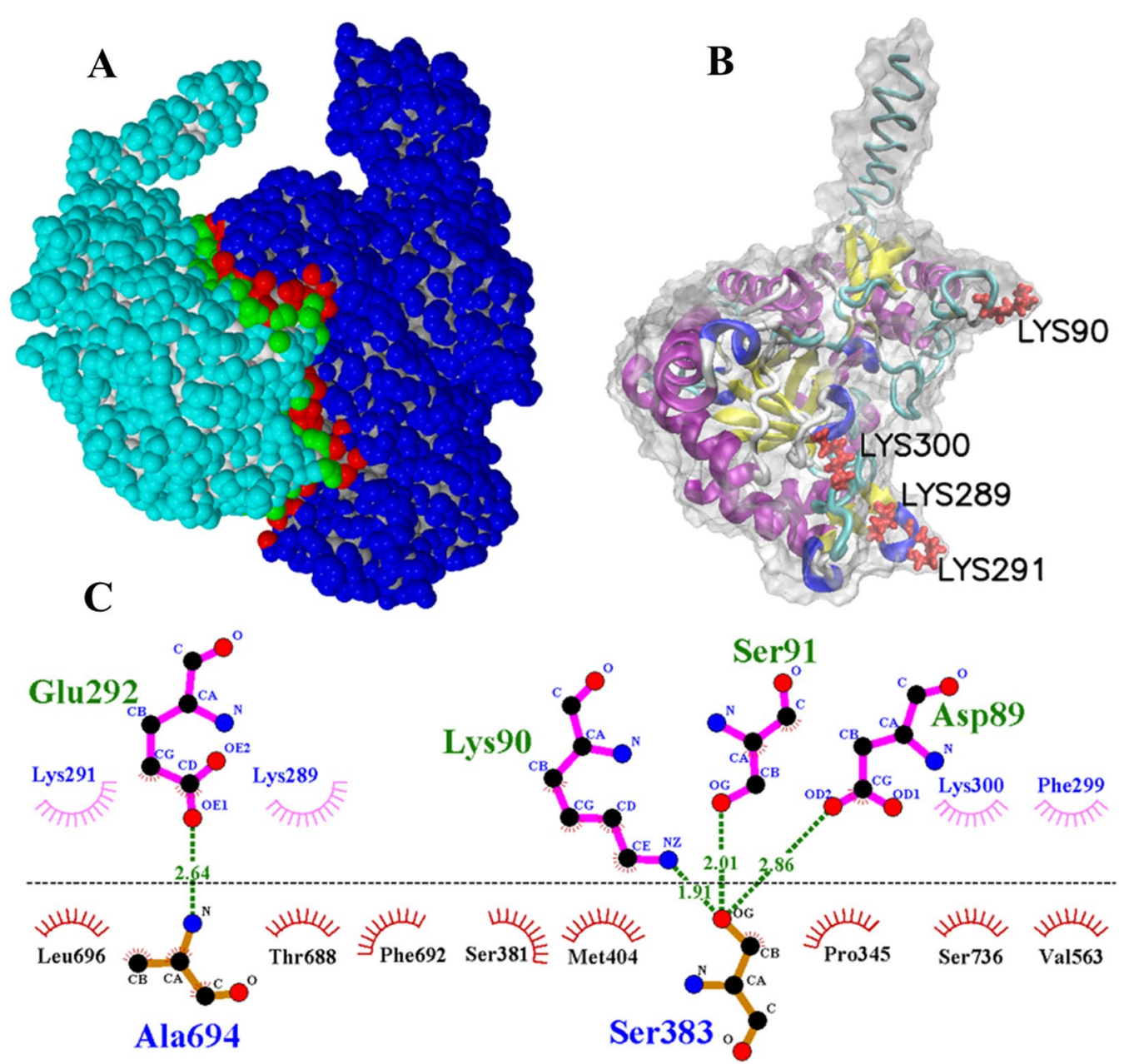

Figure 4 The interaction between TsENO and human PLG. A TSENO and PLG bound to each other at the interface region. TsENO is shown in cyan, and PLG is shown in blue. Interface-located residues of TsENO are shown in green, and those of PLG are shown in red. B The important residues (Lys90, Lys289, Lys291 and Lys300) for PLG binding are shown in red in the Corey-Pauling-Koltun (CPK) model. C TsENO-PLG interaction plot. TsENO residues are shown above the dashed line, and PLG residues are shown in different colours beneath the dashed line. Hydrogen bonds are shown as green dashed lines and labelled with the bond length $(\AA)$. The arcs represent the other residues involved in the TsENO-PLG interaction.

by SDS-PAGE and transferred to NC membranes. As confirmed by Western blot analysis, purified rTsENO, M-rTsENO and positive control ScENO were specifically recognized by the anti-PLG antibodies, whereas the negative control protein BSA was not. ML ES antigens also interacted with human PLG and showed the same binding band as rTsENO at the same position near $52 \mathrm{kDa}$. The ML soluble antigens contained many proteins that were capable of binding PLG under the same conditions. More than 10 major PLG-binding proteins, with approximate molecular masses ranging from 26.9 to $82.9 \mathrm{kDa}$, including the specific $52 \mathrm{kDa}$ band of TsENO, were observed among the ML soluble antigens (Figure 9).

\section{ELISA validation of the PLG binding and interacting residues}

The binding ELISA results confirmed that rTsENO was capable of binding human PLG and displayed an increasing trend with increasing PLG concentrations $(F=1850.78, P<0.05)$. In addition, the OD values of M-rTsENO and ScENO appeared to exhibit a dosedependent ascending pattern $\left(F_{\mathrm{M}-\mathrm{rTsENO}}=1725.65\right.$, $\left.F_{\mathrm{ScENO}}=2397.07 ; P<0.05\right)$. According to the $t$ test, the OD values of the rTsENO groups were always higher than those of the M-rTsENO groups at different PLG concentrations $\quad\left(t_{1}=40.42, \quad t_{2}=40.41, \quad t_{3}=31.84\right.$, $t_{4}=26.94, t_{5}=26.57, t_{6}=35.52, t_{7}=25.13, t_{8}=41.63$; $P<0.05)$. Compared with rTsENO, M-rTsENO showed a marked loss of PLG binding ability, with a decrease 

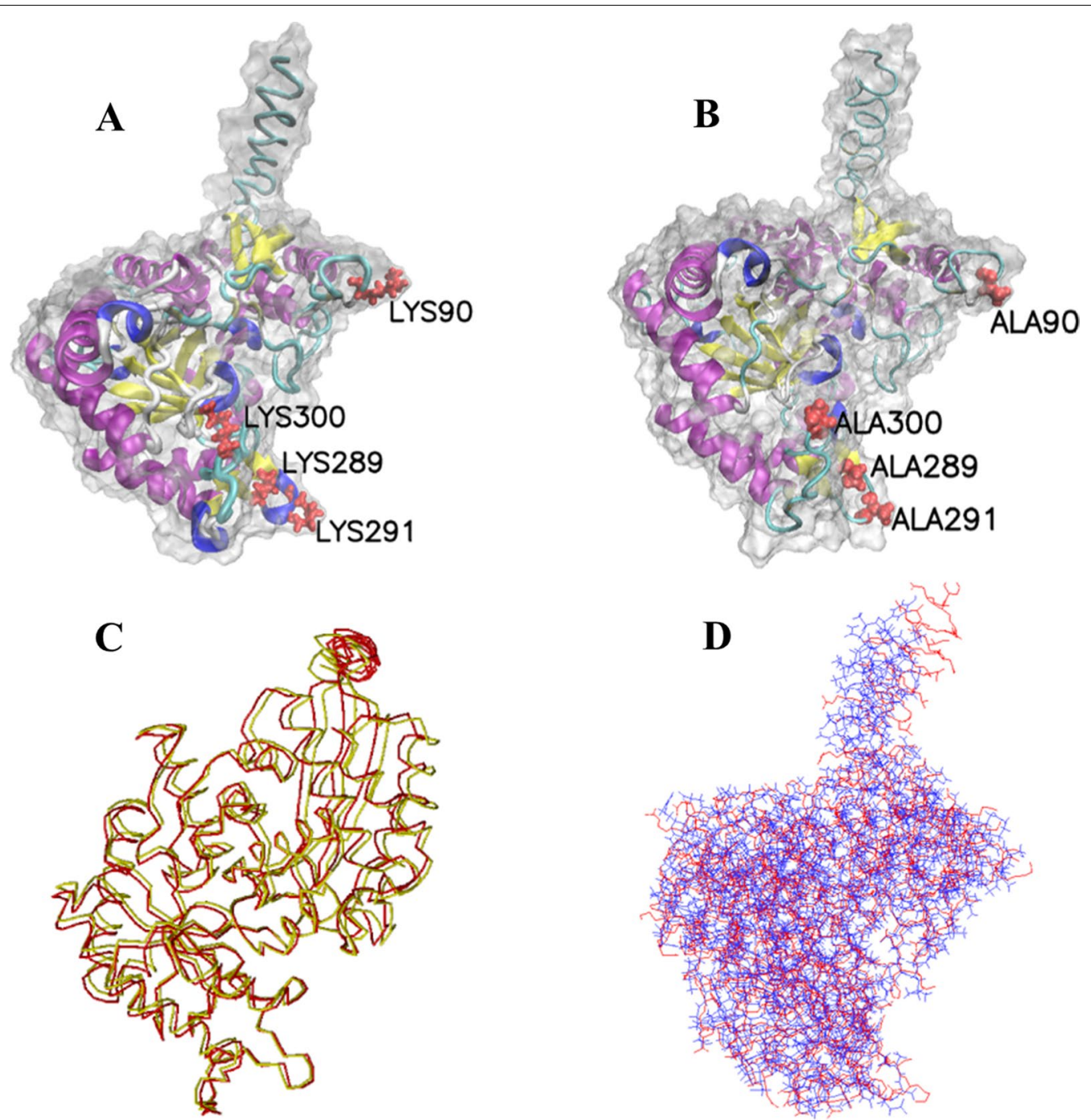

Figure 5 Structural alignments between TsENO and M-TsENO. A The interacting residues (Lys90, Lys289, Lys291 and Lys300) of wild-type TsENO are shown in red in the CPK model. B Molecular model of M-TsENO constructed by I-TASSER. The site-directed mutated residues (Ala90, Ala289, Ala291 and Ala300) of M-TsENO are shown in red in the CPK model. C Superposition of the TsENO (green) and M-TsENO (red) backbones. A local RMSD of $2.63 \AA$ A was calculated when aligned over 473 residues. D Global 3D structural alignments between TsENO (blue) and M-TsENO (red) showed a global RMSD of $3.30 \AA$.

ranging from $20.04 \%$ to $45.37 \%$. Notably, the quadruple mutant (Lys90Ala + Lys289Ala + Lys291Ala + Lys3 00Ala) M-rTsENO showed a decrease in binding with human PLG of nearly $45.37 \%$ when the concentration of PLG was $0.05 \mu \mathrm{g} / \mathrm{mL}$. Non-significant differences between $\mathrm{rTsENO}$ and ScENO were detected at lower PLG concentrations $(0.05,0.1$ and $0.2 \mu \mathrm{g} / \mathrm{mL})$. However, the binding ability of ScENO was slightly stronger than that of rTsENO at higher PLG concentrations (0.4, $0.8,1.2,1.6,2.0 \mu \mathrm{g} / \mathrm{mL})$. BSA showed no interaction with human PLG under the same experimental conditions (Figure 10).

\section{Discussion}

Enolase, also known as phosphopyruvate hydratase, was discovered in 1934 by Lohman and Mayerhof [46]. It is a pivotal enzyme in glycolysis that catalyses the dehydration of 2-phosphoglycerate (2-PGA) to phosphoenolpyruvate (PEP). While enolase is a ubiquitous metalloenzyme involved in glycolysis, it also seems to be an abundantly expressed cytosolic protein in many organisms [20,47]. Thus, the transcription of Ts-eno was observed throughout the whole life cycle of T. spiralis. Previous studies have shown that both the tricarboxylic acid cycle (Krebs cycle) and glycolysis exist in $T$. 

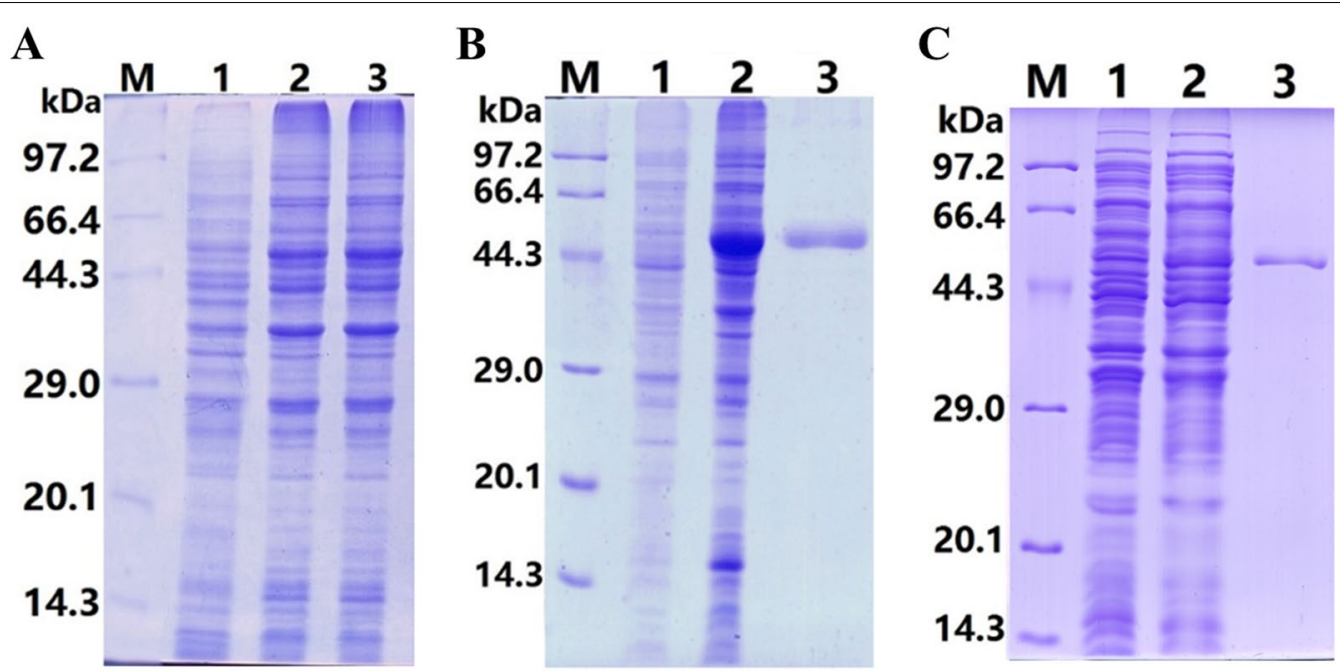

Figure 6 Expression and purification of rTsENO and M-rTsENO. A SDS-PAGE of rTsENO and M-TsENO expressed by recombinant plasmids. M: protein marker; 1: uninduced recombinant bacterial lysate; 2: induced recombinant pQE-80L/TsENO; 3: induced recombinant pQE-80L/M-TsENO. B SDS-PAGE of purified rTSENO. M: protein marker; 1: uninduced recombinant bacterial lysate; 2: induced recombinant pQE-80L/TsENO; 3: purified rTsENO $(6 \mu \mathrm{g})$. C SDS-PAGE of purified M-rTsENO. M: protein marker; 1: uninduced recombinant bacterial lysate; 2 : induced recombinant pQE-80L/ M-TsENO; 3: purified M-rTsENO $(2 \mu \mathrm{g})$.
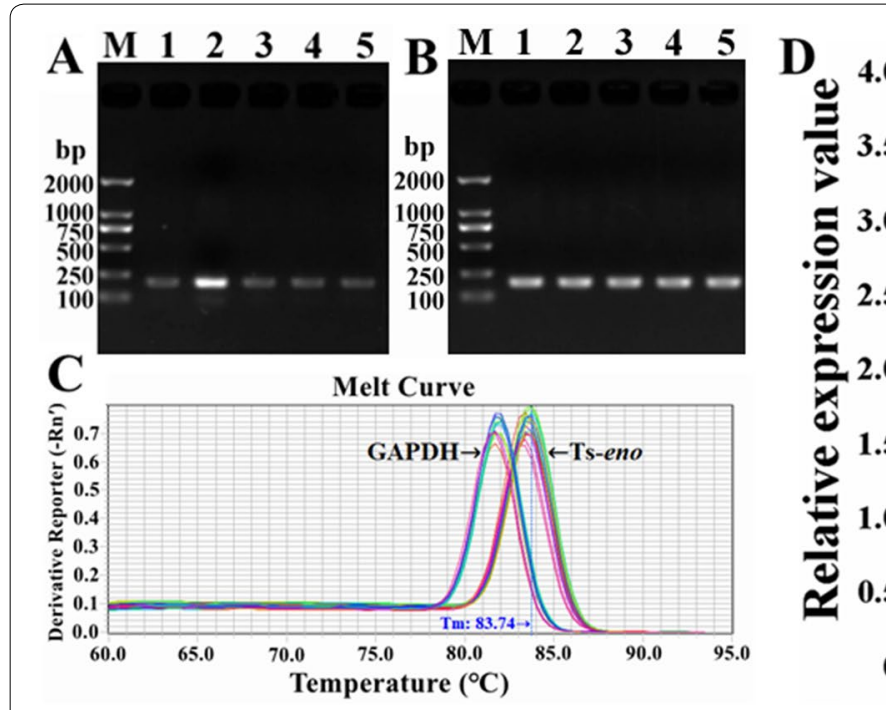

Figure 7 Real-time quantitative PCR (qPCR) analysis of Ts-eno transcription at different stages of $T$. spiralis development. A Agarose gel electrophoresis of the Ts-eno amplicon (188 bp). M: DL2000 DNA marker; 1: NBL; 2: ML; 3: IIL; 4: 3 days AW; 5: 6 days AW. B Agarose gel electrophoresis of the GAPDH amplicon (196 bp). M: DL2000 DNA marker; 1: NBL; 2: ML; 3: IIL; 4: 3 days AW; 5: 6 days AW. C Melt curves of Ts-eno and GAPDH generated by 7500 Software (version 2.0.5). D qPCR analysis of Ts-eno transcription at different T. spiralis stages. The asterisks (*) represent significant differences with other stages $(P<0.05)$.

spiralis AW and ML $[48,49]$. However, the most important metabolic pathway in ML seems to be an improved approach to classical glycolysis [50, 51]. Much more TsENO is used by ML because ML reside in the capsule in an anaerobic environment. In fact, the relative
Ts-eno transcription level was significantly higher in ML than in the other phases.

Unlike the expression and subcellular localization of other housekeeping glycolytic enzymes, the expression and subcellular localization of enolase have been found 

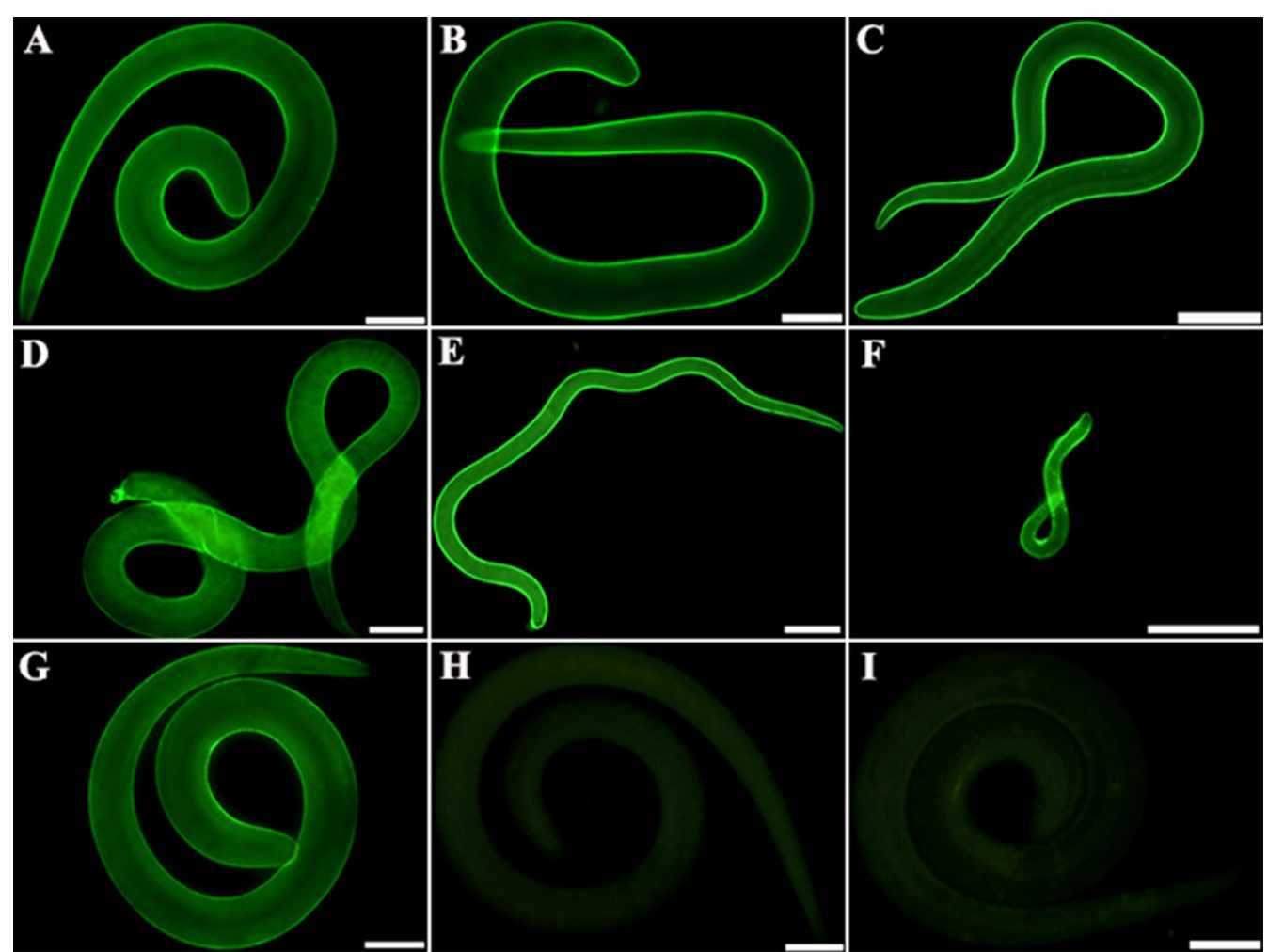

Figure 8 Immunolocalization of TsENO at different stages of T. spiralis development. A-F IFA with intact worms detected by anti-rTsENO serum. There was bright fluorescence staining on the cuticles of ML (A), $6 \mathrm{~h} \mathrm{IIL} \mathrm{(B),} 24 \mathrm{~h} \mathrm{IIL} \mathrm{(C),} 3$ days AW (D), 6 days AW (E) and NBL (F). G ML detected by infectious serum served as the positive control. H-I ML detected by PBS and normal mouse serum were used as negative controls. Scale bars: $50 \mu \mathrm{m}$.

to vary under various pathophysiological conditions [20]. In addition to its classical glycolytic enzyme function, enolase appears to be located on the surface of different kinds of pathogens, where it serves as a PLG receptor, concentrating PLM activity and facilitating invasion [14, $18,20,21]$. It has been shown that a strong relationship exists between enolase and the host's fibrinolytic system during invasion [18]. The binding between the host's PLG and helminth enolases has been studied in recent years, and the results have indicated that the PLG-enolase interaction requires lysine residues [52]. Full-length PLG comprises seven domains: $\mathrm{N}$-terminal Pan-apple domain (PA), kringle domains 1-5 (KR 1-5) and serine protease domain (SP) [8]. Interactions of the host's PLG or its isolated kringle domains with lysine residues occur in KR 1 , 2, 4 and 5 [7]. Specific structural domains named lysine binding sites (LBS) are present in these kringle domains and provide binding sites for lysine residues of PLG receptors [6]. KR 1 and KR 4 possess the highest binding affinity for lysine-type ligands [53-56], while KR 2 exhibits the lowest affinity [57].

One possible mechanism for the enhanced PLG activation rates is that the LBS within KRs interact with lysine residues of proteins on pathogen surfaces to induce PLG to adopt a conformation that can be activated [7]. Hence, the lysine residues of receptors are essential for the initial recruitment and subsequent conformational change of PLG $[8,9]$. Furthermore, recombinant enolase showed stable PLG binding and activating activity in some previous studies, indicating that enolase could play an important role in parasite invasion [58, 59]. For instance, enolases have also been reported as PLG receptors in the helminths Onchocerca volvulus, Dirofilaria immitis, Schistosoma mansoni, Schistosoma bovis, Fasciola hepatica, Echinostoma caproni, Taenia solium, Taenia multiceps and Taenia pisiformis [16, 58-65]. Among these enolases mentioned above, the enolases of Schistosoma mansoni and Taenia pisiformis were also demonstrated to promote PLG activation [16, 62]. Hence, TsENO may also play such a role in $T$. spiralis invasion by interacting with the host's PLG.

Molecular docking analysis has not been carried out for the TsENO-PLG interaction in T. spiralis. To determine the TsENO-PLG binding sites in detail, the I-TASSER program was adopted to construct the 3D structures of TsENO and its mutant in the present study. 


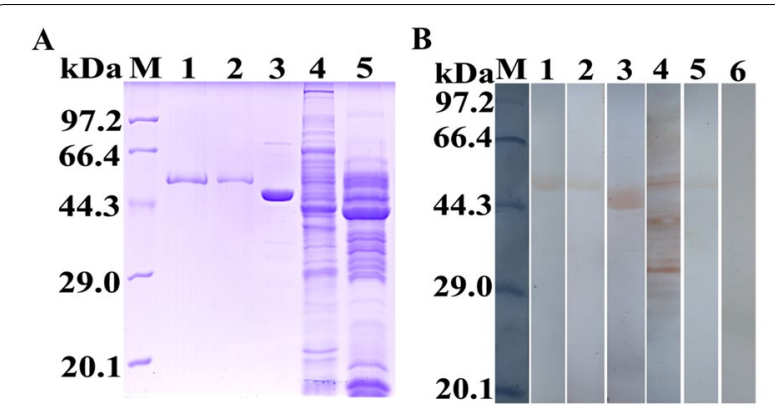

Figure 9 Western blot analysis of rTsENO and M-rTsENO binding to human PLG. A SDS-PAGE of rTsENO, M-rTsENO, ScENO, soluble antigens of ML and ES antigens of ML. M: protein marker; 1: rTsENO; 2: M-rTsENO; 3: ScENO; 4: soluble antigens of ML; 5: ES antigens of ML. B Western blot analysis of TsENO and M-rTsENO binding to human PLG. M: protein marker; 1: rTsENO; 2: M-rTsENO; 3: ScENO; 4: soluble antigens of $\mathrm{ML} ; 5$ : $\mathrm{ES}$ antigens of $\mathrm{ML} ; 6$ : BSA. The proteins mentioned above were blotted on a NC membrane and pre-incubated with human PLG. Subsequently, the membrane was incubated with an anti-PLG antibody. ScENO and BSA served as the positive control and negative control, respectively. The bands representing PLG binding to rTsENO (52 kDa), M-rTsENO (52 kDa), ScENO (47 kDa), soluble antigens of $\mathrm{ML}(26.9-82.9 \mathrm{kDa}$, including $52 \mathrm{kDa}$ ) and $\mathrm{ES}$ antigens of ML (52 kDa) were observed on the strips. The band representing PLG binding of rTsENO was more distinct than that of M-rTsENO, although the loaded amount of proteins was consistently $20 \mu \mathrm{g}$. There were some other unnamed PLG binding proteins with molecular masses ranging from 26.9 to $82.9 \mathrm{kDa}$ among $\mathrm{ML}$ soluble antigens.

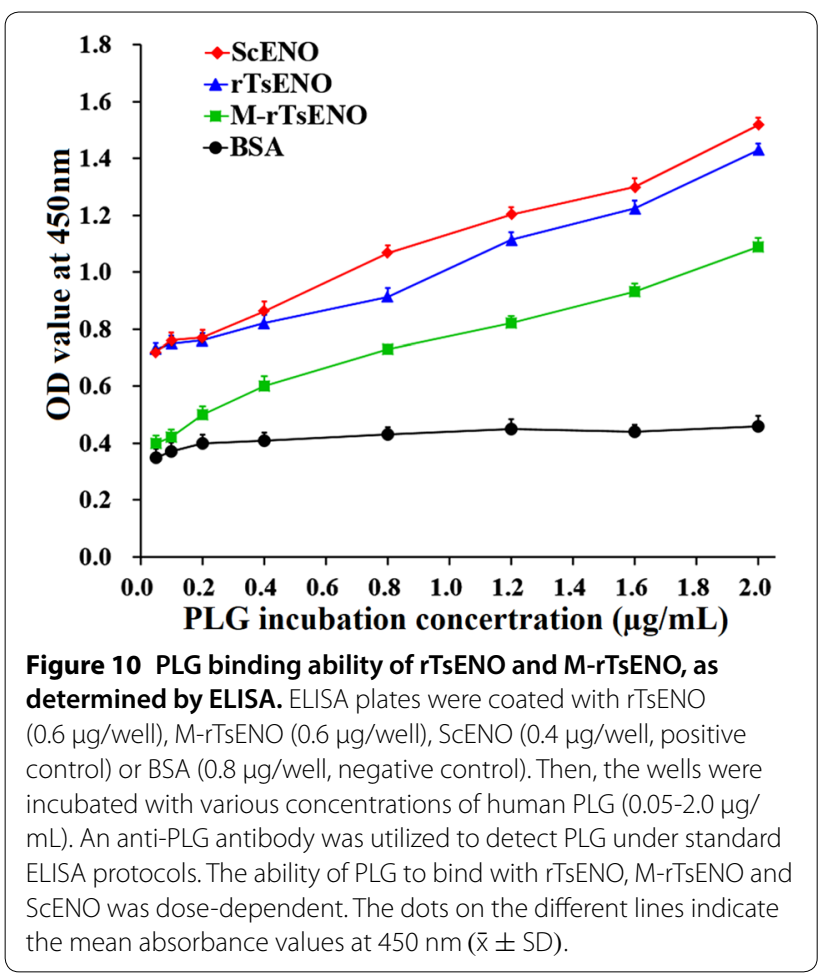

Moreover, a series of bioinformatics software programs were applied to analyse the key residues involved in the interface between TsENO and PLG. Interestingly, lysine residues were the most frequently present amino acids on the binding surface. Although Lys198, Lys229 and Lys 233 were identified in the interface, they were not the key residues for PLG binding, because the ASA loss for Lys198 was only $6.95 \AA^{2}$, which was less than $10 \AA^{2}$ [66]. In contrast, the PLG residues interacting with Lys229 and Lys 233 were located in KR 3. Considering the lack of LBS in KR 3, Lys229 and Lys233 should not be the key residues for PLG binding $[6,7,53-57]$. Notably, one of the key lysine residues (90) binds to Ser383 (located in KR 4 of PLG) by forming hydrogen bonds. The other three key lysine residues $(289,291$ and 300$)$ were also involved in hydrophobic contacts with KR 4 and the SP of PLG. These four lysine residues (90, 289, 291 and 300) of TsENO were considered to be active residues for PLG interaction. Then, we confirmed the bioinformatics prediction using site-directed mutagenesis by substituting lysine residues with neutrally charged alanines. Although the 3D structural alignments identified only slight differences between TsENO and its quadruple mutant, the decrease in PLG binding activity was significant. Hence, the reduced binding ability can be attributed to the substitution of four key lysine residues (Lys90Ala, Lys289Ala, Lys291Ala and Lys300Ala). As observed in the ELISA, the TsENO mutant lost almost half (45.37\%) of its binding ability, suggesting that these lysine residues might be crucial in the TsENO-PLG interaction.

In summary, we identified TsENO as a PLG receptor based on the results of computational methods and experimental techniques. The qPCR results showed that the Ts-eno transcription level in ML was significantly higher than in the other stages. The IFA results revealed that TsENO is expressed on the cuticle surface of T. spiralis throughout its life cycle. Furthermore, TsENO was identified by Western blotting among ES antigens of ML, consistent with the signal peptide prediction. These findings - the Ts-eno transcription level in ML and the presence of TsENO among ES antigens of ML-were somewhat different from those of one previous study on TsENO [67]. The varying lengths of TsENO sequences adopted in different studies may account for this diversity. In view of the unique mode of metabolism in the ML stage [48-51] and the protective immunity induced by enolase [68], the results of this study are still reliable and helpful for understanding the relationship between T. spiralis and its host.

Our results indicated that TsENO was capable of contact with the host's PLG either expressed on the body surface of the worm or presented in the ES products. Previous studies have shown that helminth enolases 
enhanced the activation of the host's PLG and that PLGmediated proteolysis contributed to larval invasion and migration [14-21, 58-65]; hence, TsENO could accelerate PLG activation and T. spiralis invasion of the host's intestinal wall. Unfortunately, the molecular mechanism of PLG activation by enolase and subsequent degradation of ECM is still unclear. Further studies based on in vitro and in vivo experiments should be carried out to elucidate this interesting function of TsENO in the process of larval invasion.

In conclusion, our results revealed that TsENO has strong binding activity with the host's PLG. Four lysine residues (90, 289, 291 and 300) of TsENO play an important role in PLG binding and could accelerate PLG activation and T. spiralis invasion of the host.

\section{Abbreviations}

AW: adult worms; BSA: bovine serum albumin; 3D: three-dimensional; ECM: extracellular matrix; ELISA: enzyme-linked immunosorbent assay; ES: excretory-secretory; FITC: fluorescein isothiocyanate; HRP: horseradish peroxidase; IFA: immunofluorescence assay; IIL: intestinal infective larvae; IPTG: isopropyl $\beta$-D-1-thiogalactopyranoside; I-TASSER: Iterative Threading ASSEmbly Refinement; KR: kringle domain; LBS: lysine binding sites; ML: muscle larvae; M-rTsENO: mutant recombinant T. spiralis enolase; M-TsENO: mutant T. spiralis enolase; NBL: newborn larvae; NC: nitrocellulose; OD: optical density; PBS: phosphate-buffered saline; PBST: phosphate-buffered saline containing tween; PDB: protein data bank; PLG: plasminogen; PLM: plasmin; RMSD: root mean square deviation; rTsENO: recombinant T. spiralis enolase; ScENO: S. cerevisiae enolase; SD: standard deviation; SDS-PAGE: sodium dodecyl sulfate-polyacrylamide gel electrophoresis; SP: serine protease domain; TBST: tris-buffered saline containing Tween; Ts-eno: T. spiralis enolase gene; TsENO: T. spiralis enolase.

\section{Acknowledgements}

This study was supported by grants from the National Natural Science Foundation of China (81702022) and the Training Program for Young Key Teachers of Zhengzhou University and Henan Province (2018zzu-ggjs015 and 2019HN-GGJS022)

\section{Authors' contributions}

PJ, ZQW and JC designed this study. PJ, YJZ, SWY, YYS, DMY, LYD, RDL and $X Z$ performed the experiments. PJ analysed the data with the assistance of SWY, YJZ and YYS. PJ, ZQW and JC wrote the manuscript. All authors read and approved the final manuscript.

\section{Ethics approval and consent to participate}

The use of experimental animals in this study was approved by the Life Science Ethics Committee of Zhengzhou University (No. SCXK 2015-0005).

\section{Competing interests}

The authors declare that they have no competing interests.

Received: 2 October 2019 Accepted: 8 November 2019

Published online: 05 December 2019

\section{References}

1. Cesarman-Maus G, Hajjar KA (2005) Molecular mechanisms of fibrinolysis. Br J Haematol 129:307-321

2. Urano T, Castellino FJ, Suzuki Y (2018) Regulation of plasminogen activation on cell surfaces and fibrin. J Thromb Haemost 16:1487-1497

3. Raum D, Marcus D, Alper CA, Levey R, Taylor PD, Starzl TE (1980) Synthesis of human plasminogen by the liver. Science 208:1036-1037
4. Zhang L, Seiffert D, Fowler BJ, Jenkins GR, Thinnes TC, Loskutoff DJ, Parmer RJ, Miles LA (2002) Plasminogen has a broad extrahepatic distribution. Thromb Haemost 87:493-501

5. Draxler DF, Medcalf RL (2015) The fibrinolytic system-more than fibrinolysis? Transfus Med Rev 29:102-109

6. Aisina RB, Mukhametova LI (2014) Structure and function of plasminogen/plasmin system. Russ J Bioorg Chem 40:590-605

7. Castellino FJ, Ploplis VA (2005) Structure and function of the plasminogen/plasmin system. Thromb Haemost 93:647-654

8. Law RH, Abu-Ssaydeh D, Whisstock JC (2013) New insights into the structure and function of the plasminogen/plasmin system. Curr Opin Struct Biol 23:836-841

9. Miles LA, Parmer RJ (2013) Plasminogen receptors: the first quarter century. Semin Thromb Hemost 39:329-337

10. Rahi A, Dhiman A, Singh D, Lynn AM, Rehan M, Bhatnagar R (2018) Exploring the interaction between Mycobacterium tuberculosis enolase and human plasminogen using computational methods and experimental techniques. J Cell Biochem 119:2408-2417

11. Figuera L, Gomez-Arreaza A, Avilan L (2013) Parasitism in optima forma: exploiting the host fibrinolytic system for invasion. Acta Trop 128:116-123

12. Sanderson-Smith ML, De Oliveira DM, Ranson M, McArthur JD (2012) Bacterial plasminogen receptors: mediators of a multifaceted relationship. J Biomed Biotechnol 2012:272148

13. Didiasova M, Wujak L, Wygrecka M, Zakrzewicz D (2014) From plasminogen to plasmin: role of plasminogen receptors in human cancer. Int J Mol Sci 15:21229-21252

14. Pancholi V, Chhatwal GS (2003) Housekeeping enzymes as virulence factors for pathogens. Int J Med Microbiol 293:391-401

15. Xolalpa W, Vallecillo AJ, Lara M, Mendoza-Hernandez G, Comini M, Spallek R, Singh M, Espitia C (2007) Identification of novel bacterial plasminogenbinding proteins in the human pathogen Mycobacterium tuberculosis. Proteomics 7:3332-3341

16. Zhang S, Guo A, Zhu X, You Y, Hou J, Wang Q, Luo X, Cai X (2015) Identification and functional characterization of alpha-enolase from Taenia pisiformis metacestode. Acta Trop 144:31-40

17. Siqueira GH, Atzingen MV, de Souza GO, Vasconcellos SA, Nascimento AL (2016) Leptospira interrogans Lsa23 protein recruits plasminogen, factor $\mathrm{H}$ and $\mathrm{C} 4 \mathrm{BP}$ from normal human serum and mediates $\mathrm{C} 3 \mathrm{~b}$ and $\mathrm{C} 4 \mathrm{~b}$ degradation. Microbiology 162:295-308

18. Ayon-Nunez DA, Fragoso G, Bobes RJ, Laclette JP (2018) Plasminogenbinding proteins as an evasion mechanism of the host's innate immunity in infectious diseases. Biosci Rep 38:BSR20180705

19. Hsiao KC, Shih NY, Fang HL, Huang TS, Kuo CC, Chu PY, Hung YM, Chou SW, Yang YY, Chang GC, Liu KJ (2013) Surface alpha-enolase promotes extracellular matrix degradation and tumor metastasis and represents a new therapeutic target. PLoS One 8:e69354

20. Diaz-Ramos A, Roig-Borrellas A, Garcia-Melero A, Lopez-Alemany R (2012) alpha-Enolase, a multifunctional protein: its role on pathophysiological situations. J Biomed Biotechnol 2012:156795

21. Grossi G, Grimaldi A, Cardone RA, Monne M, Reshkin SJ, Girardello R, Greco MR, Coviello E, Laurino S, Falabella P (2016) Extracellular matrix degradation via enolase/plasminogen interaction: evidence for a mechanism conserved in Metazoa. Biol Cell 108:161-178

22. Sun GG, Ren HN, Liu RD, Song YY, Qi X, Hu CX, Yang F, Jiang P, Zhang X, Wang ZQ, Cui J (2018) Molecular characterization of a putative serine protease from Trichinella spiralis and its elicited immune protection. Vet Res 49:59

23. Song YY, Wang LA, Na Ren H, Qi X, Sun GG, Liu RD, Jiang P, Zhang X, Cui J, Wang ZQ (2018) Cloning, expression and characterisation of a cysteine protease from Trichinella spiralis. Folia Parasitol (Praha) 65:2018.007

24. Liu CY, Ren HN, Song YY, Sun GG, Liu RD, Jiang P, Long SR, Zhang X, Wang ZQ, Cui J (2018) Characterization of a putative glutathione S-transferase of the parasitic nematode Trichinella spiralis. Exp Parasitol 187:59-66

25. Wang L, Wang ZQ, Cui J (2013) Proteomic analysis of the changed proteins of Trichinella spiralis infective larvae after co-culture in vitro with intestinal epithelial cells. Vet Parasitol 194:160-163

26. Liu RD, Wang ZQ, Wang L, Long SR, Ren HJ, Cui J (2013) Analysis of differentially expressed genes of Trichinella spiralis larvae activated by bile and cultured with intestinal epithelial cells using real-time PCR. Parasitol Res 112:4113-4120 
27. Liu RD, Cui J, Liu XL, Jiang P, Sun GG, Zhang X, Long SR, Wang L, Wang ZQ (2015) Comparative proteomic analysis of surface proteins of Trichinella spiralis muscle larvae and intestinal infective larvae. Acta Trop 150:79-86

28. Gasteiger E, Hoogland C, Gattiker A, Duvaud S, Wilkins MR, Appel RD, Bairoch A (2005) Protein identification and analysis tools on the ExPASy server. In: Walker JM (ed) The proteomics protocols handbook. Humana Press, Totowa, pp 571-607

29. Petersen TN, Brunak S, von Heijne G, Nielsen H (2011) SignalP 4.0: discriminating signal peptides from transmembrane regions. Nat Methods 8:785-786

30. Yang J, Yan R, Roy A, Xu D, Poisson J, Zhang Y (2015) The I-TASSER suite: protein structure and function prediction. Nat Methods 12:7-8

31. Laskowski RA, Rullmannn JA, MacArthur MW, Kaptein R, Thornton JM (1996) AQUA and PROCHECK-NMR: programs for checking the quality of protein structures solved by NMR. J Biomol NMR 8:477-486

32. Lüthy R, Bowie JU, Eisenberg D (1992) Assessment of protein models with three-dimensional profiles. Nature 356:83-85

33. Colovos C, Yeates TO (1993) Verification of protein structures: patterns of nonbonded atomic interactions. Protein Sci 2:1511-1519

34. Hooft RW, Vriend G, Sander C, Abola EE (1996) Errors in protein structures. Nature 381:272

35. Pontius J, Richelle J, Wodak SJ (1996) Deviations from standard atomic volumes as a quality measure for protein crystal structures. J Mol Biol 264:121-136

36. Pierce BG, Wiehe K, Hwang H, Kim BH, Vreven T, Weng Z (2014) ZDOCK server: interactive docking prediction of protein-protein complexes and symmetric multimers. Bioinformatics 30:1771-1773

37. Humphrey W, Dalke A, Schulten K (1996) VMD: visual molecular dynamics. J Mol Graph 14:33-38

38. Laskowski RA, Swindells MB (2011) LigPlot+: multiple ligand-protein interaction diagrams for drug discovery. J Chem Inf Model 51:2778-2786

39. Krissinel E, Henrick K (2007) Inference of macromolecular assemblies from crystalline state. J Mol Biol 372:774-797

40. Maiti R, Van Domselaar GH, Zhang H, Wishart DS (2004) SuperPose: a simple server for sophisticated structural superposition. Nucleic Acids Res 32:W590-W594

41. Guex N, Peitsch MC (1997) SWISS-MODEL and the Swiss-PdbViewer: an environment for comparative protein modeling. Electrophoresis 18:2714-2723

42. Qi X, Yue $X$, Han Y, Jiang P, Yang F, Lei JJ, Liu RD, Zhang X, Wang ZQ, Cui J (2018) Characterization of two Trichinella spiralis adult-specific DNase II and their capacity to induce protective immunity. Front Microbiol 9:2504

43. Schmittgen TD, Livak KJ (2008) Analyzing real-time PCR data by the comparative CT method. Nat Protoc 3:1101-1108

44. Sun GG, Song YY, Jiang P, Ren HN, Yan SW, Han Y, Liu RD, Zhang X, Wang ZQ, Cui J (2018) Characterization of a Trichinella spiralis putative serine protease. Study of its potential as sero-diagnostic tool. PLoS Negl Trop Dis 12:e0006485

45. Xu J, Yang F, Yang DQ, Jiang P, Liu RD, Zhang X, Cui J, Wang ZQ (2018) Molecular characterization of Trichinella spiralis galectin and its participation in larval invasion of host's intestinal epithelial cells. Vet Res 49:79

46. Ji H, Wang J, Guo J, Li Y, Lian S, Guo W, Yang H, Kong F, Zhen L, Guo L, Liu $Y(2016)$ Progress in the biological function of alpha-enolase. Anim Nutr 2:12-17

47. Pancholi V (2001) Multifunctional alpha-enolase: its role in diseases. Cell Mol Life Sci 58:902-920

48. Goldberg E (1957) Studies on the intermediary metabolism of Trichinella spiralis. Exp Parasitol 6:367-382

49. Goldberg E (1958) The glycolytic pathway in Trichinella spiralis larvae. J Parasitol 44:363-370

50. Karpiak SE, Kozar Z (1963) Changes in the metabolism of the skeletal muscles of guinea pigs caused by the invasion of Trichinella spiralis. I. Influence of the invasion on the carbohydrate metabolism of muscles. Wiad Parazytol 9:435-446

51. Ward CW, Castro GA, Fairbairn D (1969) Carbon dioxide fixation and phosphoenolpyruvate metabolism in Trichinella spiralis larvae. J Parasitol 55:67-71
52. Gonzalez-Miguel J, Morchon R, Siles-Lucas M, Oleaga A, Simon F (2015) Surface-displayed glyceraldehyde 3-phosphate dehydrogenase and galectin from Dirofilaria immitis enhance the activation of the fibrinolytic system of the host. Acta Trop 145:8-16

53. Sehl LC, Castellino FJ (1990) Thermodynamic properties of the binding of $a-, \omega$-amino acids to the isolated kringle 4 region of human plasminogen as determined by high sensitivity titration calorimetry. J Biol Chem 265:5482-5486

54. Menhart N, Sehl LC, Kelley RF, Castellino FJ (1991) Construction, expression and purification of recombinant kringle 1 of human plasminogen and analysis of its interaction with $\omega$-amino acids. Biochemistry 30:1948-1957

55. Menhart N, McCance SG, SehI LC, Castellino FJ (1993) Functional independence of the kringle 4 and kringle 5 regions of human plasminogen. Biochemistry 32:8799-8806

56. Menhart N, Castellino FJ (1995) The importance of the hydrophobic components of the binding energies in the interaction of $\omega$-amino acid ligands with isolated kringle polypeptide domains of human plasminogen. Int J Pept Protein Res 46:464-470

57. Marti DN, Schaller J, Llinás M (1999) Solution structure and dynamics of the plasminogen kringle 2-AMCHA complex: 3(1)-helix in homologous domains. Biochemistry 38:15741-15755

58. Zhang S, You Y, Luo X, Zheng Y, Cai X (2018) Molecular and biochemical characterization of Taenia solium alpha-enolase. Vet Parasitol 254:36-42

59. Ayon-Nunez DA, Fragoso G, Espitia C, Garcia-Varela M, Soberon X, Rosas G, Laclette JP, Bobes RJ (2018) Identification and characterization of Taenia solium enolase as a plasminogen-binding protein. Acta Trop 182:69-79

60. Jolodar A, Fischer P, Bergmann S, Büttner DW, Hammerschmidt S, Brattig NW (2003) Molecular cloning of an a-enolase from the human filarial parasite Onchocerca volvulus that binds human plasminogen. Biochim Biophys Acta 1627:111-120

61. Gonzalez-Miguel J, Morchon R, Carreton E, Montoya-Alonso JA, Simon F (2013) Surface associated antigens of Dirofilaria immitis adult worms activate the host fibrinolytic system. Vet Parasitol 196:235-240

62. Figueiredo BC, Da'dara AA, Oliveira SC, Skelly PJ (2015) Schistosomes enhance plasminogen activation: the role of tegumental enolase. PLoS Pathog 11:e1005335

63. Ramajo-Hernandez A, Perez-Sanchez R, Ramajo-Martin V, Oleaga A (2007) Schistosoma bovis: plasminogen binding in adults and the identification of plasminogen-binding proteins from the worm tegument. Exp Parasitol 115:83-91

64. Bernal D, de la Rubia JE, Carrasco-Abad AM, Toledo R, Mas-Coma S, Marcilla A (2004) Identification of enolase as a plasminogen-binding protein in excretory-secretory products of Fasciola hepatica. FEBS Lett 563:203-206

65. Li WH, Qu ZG, Zhang NZ, Yue L, Jia WZ, Luo JX, Yin H, Fu BQ (2015) Molecular characterization of enolase gene from Taenia multiceps. Res Vet Sci 102:53-58

66. Ghosh KS, Sen S, Sahoo BK, Dasgupta S (2009) A spectroscopic investigation into the interactions of 3'-O-carboxy esters of thymidine with bovine serum albumin. Biopolymers 91:737-744

67. Nakada T, Nagano I, Wu Z, Takahashi Y (2005) Molecular cloning and functional expression of enolase from Trichinella spiralis. Parasitol Res 96:354-360

68. Zhang X, Xu L, Song X, Li X, Yan R (2018) Molecular cloning of enolase from Trichinella spiralis and the protective immunity in mice. Acta Parasitol 63:252-260

\section{Publisher's Note}

Springer Nature remains neutral with regard to jurisdictional claims in published maps and institutional affiliations. 